\title{
Valence Surface Electronic States on Ge(001)
}

\author{
M. W. Radny, ${ }^{1, *}$ G. A. Shah, ${ }^{1}$ S. R. Schofield,,${ }^{1, \dagger}$ P. V. Smith, ${ }^{1}$ and N. J. Curson ${ }^{2}$ \\ ${ }^{1}$ School of Mathematical and Physical Sciences, The University of Newcastle, Callaghan 2308, Australia \\ ${ }^{2}$ London Centre for Nanotechnology and Department of Electronic and Electrical Engineering, University College, \\ London WC1H OAH, United Kingdom
}

(Received 6 February 2008; published 20 June 2008)

\begin{abstract}
The optical, electrical, and chemical properties of semiconductor surfaces are largely determined by their electronic states close to the Fermi level $\left(E_{F}\right)$. We use scanning tunneling microscopy and density functional theory to clarify the fundamental nature of the ground state $\mathrm{Ge}(001)$ electronic structure near $E_{F}$, and resolve previously contradictory photoemission and tunneling spectroscopy data. The highest energy occupied surface states were found to be exclusively back bond states, in contrast to the $\mathrm{Si}(001)$ surface, where dangling bond states also lie at the top of the valence band.
\end{abstract}

PACS numbers: 73.20.At, 68.37.Ef

The $\mathrm{Si}(001)$ surface is perhaps the most widely studied surface in science and is often assumed to be a model for all other (001) semiconductor surfaces. We have found, however, that the $\mathrm{Ge}(001)$ surface exhibits distinctly different electronic behavior close to the Fermi energy $\left(E_{F}\right)$. A detailed understanding of such differences is of fundamental importance [1,2], since many of the key properties of semiconductors, such as their chemical activity, and electrical and optical response, depend critically on the nature of the electronic states close to $E_{F}$. The results presented here are therefore important for the development of future atomic-scale devices based on $\mathrm{Ge}(001)$.

We present atomic resolution, filled-state biasdependent STM images of the ground state $\mathrm{Ge}(001) c(4 \times$ 2) surface. Comparison of these images with those simulated from our $a b$ initio density functional theory (DFT) calculations unambiguously shows that the states at the top of the $\mathrm{Ge}(001)$ valence band arise solely from back bonds, and that the occupied dangling bond states of the surface atoms lie $\sim 0.35 \mathrm{eV}$ lower in energy. This is contrary to the $\mathrm{Si}(001) c(4 \times 2)$ surface where both the dangling bond and back bond surface states occur together at the top of the valence band. Our results thus indicate that the $\mathrm{Ge}(001)$ surface may produce different behavior to $\mathrm{Si}(001)$ even though these two surfaces are structurally very similar.

Both $\mathrm{Si}(001)$ and $\mathrm{Ge}(001)$ surfaces reconstruct into rows of paired atoms called dimers. Formally, each $\mathrm{Si}-\mathrm{Si}$ or $\mathrm{Ge}-$ Ge dimer can be described in terms of a very strong covalent $\sigma$ bond between the dimer atoms, and a much weaker $\pi$ bond between the dangling bond orbitals of the dimer atoms, as shown in Fig. 1(a). The ground state for both surfaces consists of buckled (asymmetric) dimers [Fig. 1(b)], forming a $c(4 \times 2)$ reconstruction [3]. The charge distribution of a buckled dimer due to the $\pi-$ component is asymmetric with the charge shifting from the down-atom to the up-atom. This results in the up-atom dangling bond becoming doubly occupied, while the down-atom dangling bond is empty [Fig. 1(b)]. On the extended $\mathrm{Si}(001)$ and $\mathrm{Ge}(001) c(4 \times 2)$ surfaces, interac- tion between the dimers opens up a surface energy gap at $E_{F}$ resulting in both surfaces being semiconducting. The measured values of the energy gap are $\sim 0.7 \mathrm{eV}$ and $\sim 0.3 \mathrm{eV}$ for $\mathrm{Si}(001)$ and $\mathrm{Ge}(001)$, respectively. On $\mathrm{Si}(001)$ the gap separates the occupied $\pi$ dangling bond surface states below $E_{F}$, from the empty $\pi^{*}$ dangling bond surface states above $E_{F}$. The corresponding data for $\mathrm{Ge}(001)$, provided by both theory and experiment, however, are conflicting [4-7].

For $\mathrm{Ge}(001)$, the main controversy relates to the nature of the electronic states at the top of the valence band, close to $E_{F}$. In early photoemission studies [4], these states were observed to disappear after hydrogen chemisorption, and hence were assumed to be surface dangling bond states. Based on their very recent photoemission data Jeon et al. [6] also suggest that the states at the top of the valence band $\sim 0.3 \mathrm{eV}$ below $E_{F}$ are due to the surface dangling bonds. In a combined photoemission and STM study, however, the electronic states at the top of the Ge(001) valence band were assumed to be bulk states [7]. This assignment was based on the observation that the valence band structure remained essentially unchanged for temperatures between 130 and $680 \mathrm{~K}$, as well as being unaffected by the chemisorption of hydrogen. A recent scanning tunneling spectroscopy (STS) experiment [8] identified the dangling bond state on $\mathrm{Ge}(001) c(4 \times 2)$ as being $-0.8 \mathrm{eV}$ below $E_{F}$. The same report suggested that a feature at $-0.5 \mathrm{eV}$ could also be linked to the dangling bonds while, interestingly, a (a)

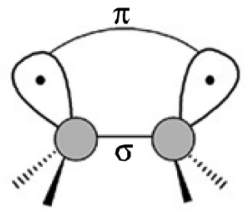

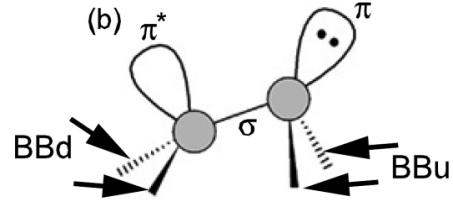

FIG. 1. Schematics of the bonding structures of (a) symmetric and (b) buckled dimers of the dimerized $\mathrm{Ge}(001)$ and $\mathrm{Si}(001)$ surfaces. The labels $\mathrm{BBd}$ and $\mathrm{BBu}$ denote the back bonds associated with the down and up dimer atoms, respectively. 
clearly visible spectral feature at $\sim-0.1 \mathrm{eV}$ was not discussed at all.

Our measurements have been carried out for the $\mathrm{Ge}(001) c(4 \times 2)$ surface at room temperature $(\mathrm{RT})$ using a commercial STM (Omicron). At RT, the buckling of the dimers of the $\mathrm{Ge}(001)$ surface alternates rapidly compared to the scan motion of the STM tip, and results in each dimer appearing as a single symmetric protrusion in STM images. To pin the surface into a $c(4 \times 2)$ configuration we have dosed an $n$-type $\mathrm{Ge}(001)$ surface lightly with $\mathrm{H}$ atoms produced by cracking $99.999 \%$ pure molecular $\mathrm{H}_{2}$ gas upon entry to the UHV chamber using a hot tungsten filament.

Figure 2(a) shows an STM image of a H-dosed surface. The $\mathrm{H}$ atoms form intrinsically buckled $\mathrm{Ge}-\mathrm{Ge}-\mathrm{H}$ hemihydride dimers (bright protrusions). These buckled defects cause the surrounding surface to lock into a particular buckling orientation. Figures 2(b)-2(e) show the H-free region of the $\mathrm{H}$-dosed $\mathrm{Ge}(001)$ surface indicated by the white rectangle in Fig. 2(a). It is understood that the bright contrast in filled-state STM images results from the electronic charge located on the up-buckled atom of each dimer. We thus interpret the $-0.6 \mathrm{~V}$ and $-0.4 \mathrm{~V}$ experimental STM images shown in Figs. 2(b) and 2(c) as revealing sets of parallel rows of tilted Ge-Ge dimers.

(a)

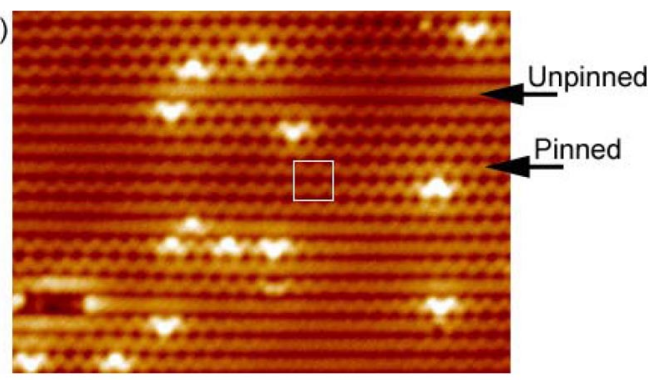

(b) $-0.6 \mathrm{~V}$ (c) $-0.4 \mathrm{~V}$ (d) $-0.2 \mathrm{~V}$ (e) $-0.1 \mathrm{~V}$
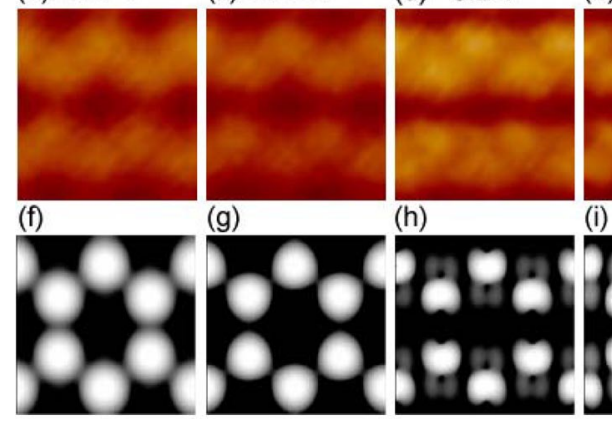
(g) (h)
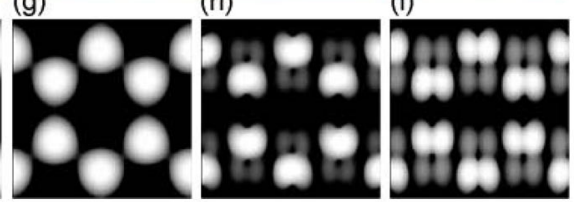

FIG. 2 (color online). Filled-state STM images of clean $\mathrm{Ge}(001)$ at sample biases of (a) $-1.0 \mathrm{~V}$, (b) $-0.6 \mathrm{~V}$, (c) $-0.4 \mathrm{~V}$, (d) $-0.2 \mathrm{~V}$, (e) $-0.1 \mathrm{~V}$. The images in (b)(e) are for the area of $\sim 2.3 \times 3.7 \mathrm{~nm}^{2}$ indicated by the white rectangle in (a). All images were acquired at $\sim 0.8 \mathrm{nA}$ tunneling current. Simulated filled-state STM images for $\mathrm{Ge}(001) c(4 \times 2)$ corresponding to an integration range of (f) $-0.6 \mathrm{eV}$, (g) $-0.4 \mathrm{eV}$, (h) $-0.3 \mathrm{eV}$, and (i) $-0.2 \mathrm{eV}$. All of these ILDOS plots are for an isosurface value of $1.8 \times 10^{-4} \mathrm{e} / \AA^{3}$.
Within each dimer row, the neighboring dimers buckle in opposite directions producing a typical $2 \times 2$ zigzag pattern. Out of phase buckling of the dimers on adjacent dimer rows produces the well known $c(4 \times 2)$ appearance characteristic of the ground state of $\mathrm{Si}(001)$ and $\mathrm{Ge}(001)$ surfaces.

In contrast to the familiar images in Figs. 2(b) and 2(c) (which were acquired at $-0.6 \mathrm{~V}$ and $-0.4 \mathrm{~V}$ ), the image shown in Fig. 2(e) for the very low sample bias of $-0.1 \mathrm{~V}$ exhibits a quite different appearance. In particular, the bright protrusions centered on the $\mathrm{Ge}$ up atoms in Figs. 2(b) and 2(c) have been replaced by double-lobed features at the same atomic positions. As the dangling bond of an up-Ge atom is mainly formed from its $4 p_{z}$ orbital, the double-lobed feature cannot originate from the dangling bond state. We will show that these low-bias voltage features originate from the back bond states of the up-Ge atoms of the buckled Ge-Ge dimers. At $-0.2 \mathrm{~V}$ sample bias [Fig. 2(d)] the appearance is intermediate between the double-lobed and dangling bond features.

The origin of the bias-dependent features observed in the STM images have been investigated by performing fully spin polarized DFT calculations [9] following the same procedure as outlined in Ref. [10]. The clean Ge(001) surface was modeled within a $(4 \times 4) 8$-dimer unit cell using $p(2 \times 2)$ and $c(4 \times 2)$ periodicities. The energy difference between these two configurations was determined to be $6 \mathrm{meV}$, with the $c(4 \times 2)$ geometry being lower in energy. This $c(4 \times 2)$ configuration is clearly evident in the STM image of Fig. 2(a) and hence is used in our analysis. The buckling angle of the Ge dimers and the dimer bond-length were calculated to be $19.6^{\circ}$ and $2.59 \AA$, respectively, in good agreement with previously published experimental data $[11,12]$.

The experimental STM images of the $\mathrm{Ge}(001) c(4 \times 2)$ surface were correlated with the calculated electronic structure by generating integrated local density of states (ILDOS) plots. Such plots provide a first order approximation to the expected appearance of the STM images [13]. Approximations to the STM images are produced by tracing a specific isocontour of the integrated surface LDOS. Such plots are shown in Figs. 2(f)-2(i) for integration ranges from $E_{F}$ of $-0.6 \mathrm{eV},-0.4 \mathrm{eV},-0.3 \mathrm{eV}$, and $-0.2 \mathrm{eV}$, which were chosen to represent the bias voltage range of the experimental images. The bright protrusions in Figs. 2(f) and 2(g) denote the high charge densities centered on the up-Ge atoms of the dimers, and reproduce the static buckling of the $\mathrm{Ge}(001) c(4 \times 2)$ surface in agreement with the experimental STM images presented in Figs. 2(b) and 2(c). The $-0.2 \mathrm{eV}$ integrated plot in Fig. 2(i) models the smallest negative bias voltage of $-0.1 \mathrm{~V}$ (we note that the surface states become accessible in experiment at a lower bias than in the DFT calculation due to tip-induced band bending). This image is seen to be in excellent agreement with the experimental filled-state 
STM image in Fig. 2(e) where each protrusion appears as a double-lobed feature. The image in Fig. 2(h) represents the transition from the double-lobed features of Fig. 2(i) to the more symmetrical features of Fig. 2(g).

To demonstrate that the double-lobed features appearing in the very low-bias images such as Fig. 2(e) originate from the back bonds, and not from the dangling bonds of the GeGe dimers, we have analyzed the surface electronic structure of the clean $\mathrm{Ge}(001) c(4 \times 2)$ surface in more detail. The surface electronic band structure in the vicinity of $E_{F}$ calculated for this surface using a $4 \times 4$ cell is shown in Fig. 3. The system is observed to be semiconducting.

Figure 4 shows charge density plots for the highest occupied surface state corresponding to the k-point lying one quarter of the way along the $\Gamma-K$ symmetry direction of the SBZ (indicated by the circle in Fig. 3). This state, which is characteristic of all of the states indicated by the filled squares in Fig. 3, is composed of both first and third layer back bonds [i.e., charge between the first and second, and third and fourth, layer atoms - see Fig. 4(c)]. Such spatial localization of charge is characteristic of a surface state in the energy gap region. As shown in Fig. 3, these back bond surface states are the only ones included within the $-0.2 \mathrm{eV}$ integration range of the simulated image of Fig. 2(i). Thus, the dominant double-lobed features in this simulated image [and in the corresponding experimental image of Fig. 2(e)] originate from the Ge dimer back bonds to the second-layer atoms.

It should be noted that the calculated charge density plots in Figs. 4(a) and 4(c) indicate that more of the charge of this back bond surface state is centered on the downatoms of the Ge-Ge dimers than on the up atoms, while the features in the experimental [Fig. 2(e)] and simulated [Fig. 2(i)] STM images are centered on the up-Ge dimer atoms. This apparent contradiction is easily explained by noting that an STM tip probes the charge distribution some distance above the surface. The charge density distribution for this back bond state above a plane some distance above

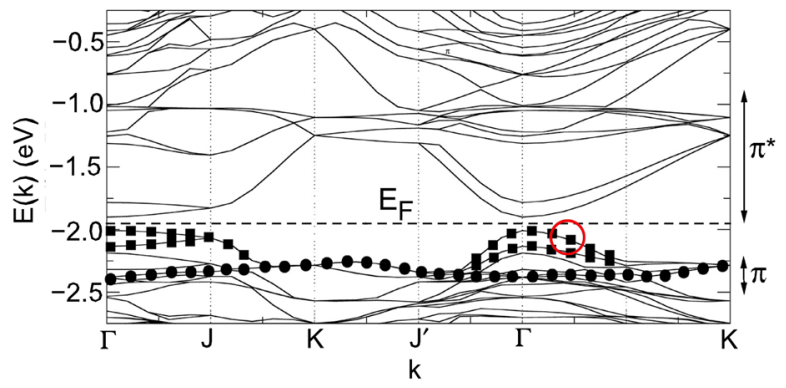

FIG. 3 (color online). The electronic structure of the clean $\mathrm{Ge}(001) c(4 \times 2)$ surface close to $E_{F}$ (dashed horizontal line) calculated using a $(4 \times 4)$ surface unit cell. The filled squares denote the surface back bonds, and the filled circles indicate the $\pi$ dangling bond surface states. The unoccupied $\left(\pi^{*}\right)$ dangling bond surface states are indicated by the double-headed arrow above $E_{F}$. the surface dimers is plotted in Fig. 4(b) for the isosurface value of $3.8 \times 10^{-4} \mathrm{e} / \AA^{3}$. This plot closely mirrors our simulated STM image in Fig. 2(i) and shows significant charge in the vacuum region associated with only the upGe atoms of the dimers. This is due to the buckling of the dimers which ensures that the charge distribution of the back bonds associated with the up dimer atoms extends further into the vacuum than the more horizontal back bonds involving the down dimer atoms [see Fig. 4(c)].

The dangling bond states of the Ge dimer atoms are indicated by the filled circles in Fig. 3 and constitute a narrow $\pi$ band located $\sim 0.35 \mathrm{eV}$ below $E_{F}$. These states have their charge localized primarily on the up-atoms of the Ge-Ge dimers [Fig. 1(b)]. Around the $J$ point, and along parts of the $\Gamma-J^{\prime}$ and $\Gamma-K$ symmetry directions, these states are energetically resonant with the bulk states and are no longer exclusively localized in the surface region as is the case for the back bond states [see Fig. 4(c)]. Integrating the LDOS over a range of $-0.4 \mathrm{eV}$ (or more) from the Fermi energy results in the inclusion of both the back bond states and some of the dangling bond surface states (see Fig. 3). The latter have relatively large charge densities above the surface and hence they dominate over the back bond states in the STM appearance of the Ge(001) surface at higher negative bias voltage [Figs. 2(b) and 2(c)]. This is clearly evidenced by the $-0.4 \mathrm{eV}$ integrated image in Fig. 2(g) where the double-lobed features of Fig. 2(i) are replaced by more symmetrical features like those in Figs. 2(f).

The above results resolve earlier ambiguous experimental data regarding the $\mathrm{Ge}(001) c(4 \times 2)$ surface. In particular, our results indicate that the clearly visible but unassigned STS spectral feature at $\sim-0.1 \mathrm{~V}$ seen by Gurlu et al. for $\mathrm{Ge}(001) c(4 \times 2)$ (see Fig. 2 of Ref. [8]) arises from the back bond surface states. The observed low intensity of this spectral peak results from the significant

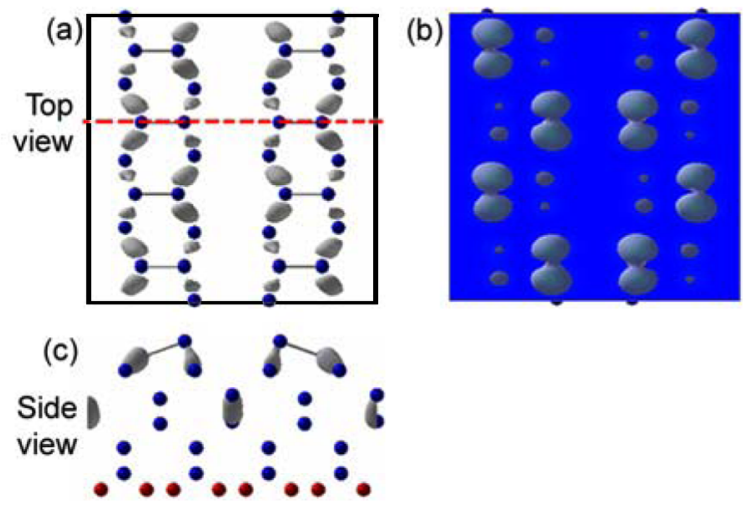

FIG. 4 (color online). Charge density plots for the highest occupied state at the k-point one quarter of the distance along the $\Gamma-K$ symmetry direction (see Fig. 3); (a) top, and (c) side views (isosurface value $6.5 \times 10^{-3} \mathrm{e} / \AA^{3}$ ); (b) plane above the $\mathrm{Ge}(001) c(4 \times 2)$ surface (isosurface value $\left.3.8 \times 10^{-4} \mathrm{e} / \AA^{3}\right)$. The plot in (c) is along the dashed line in (a). 
dispersion of these states around the $\Gamma$ point. We have also performed calculations (not presented here) on the fully $\mathrm{H}$ terminated $\mathrm{Ge}(001)$ surface which consists of symmetric Ge dimers. These calculations have shown that, while the saturated dangling bond states are shifted well away from the Fermi energy, the chemisorption of $\mathrm{H}$ does not significantly affect the energy of the back bond states. The earlier assignment of such $\mathrm{H}$ independent states to the bulk states [7] is thus not warranted. In addition, we have calculated the band structure for the symmetric dimer Ge(001) $2 \times 1$ surface. These calculations have revealed that the energies of the back bond states are little affected by the transformation from buckled $c(4 \times 2)$ to symmetric $(2 \times 1)$ dimers. However, the dangling bond states on the $(2 \times 1)$ surface are shifted to the Fermi level, resulting in the surface being metallic. This result agrees with recent STS measurements [8] where local phases of $c(4 \times 2)$ and $(2 \times 1)$ reconstruction were reported to be semiconducting and metallic, respectively.

The differences in the nature and location of the states close to $E_{F}$ of the $(2 \times 1)$ and $c(4 \times 2) \mathrm{Ge}(001)$ surfaces should result in these two surfaces behaving quite differently. Many adsorbates (e.g., $\mathrm{Cl}, \mathrm{H}, \mathrm{O}$ ) interact with a dimerised surface such as $\mathrm{Ge}(001)$ by accepting charge from the surface dangling bond states lying close to $E_{F}$. For the symmetric dimer Ge(001) surface, which models the rapid flip-flopping of the buckled dimers at elevated temperatures, these dangling bond states lie near $E_{F}$. For the $\mathrm{Ge}(001) c(4 \times 2)$ surface, however, the states close to $E_{F}$ are the back bond states which have very different charge distributions and orbital symmetry. Within the frontier orbital picture of molecular bonding [14] this suggests that the chemistry (adsorption, surface diffusion, etc.) of the $\mathrm{Ge}(001)$ surface may be quite sensitive to factors such as temperature and defects. We note that a very low density of surface defects on $\mathrm{Ge}(001)$ ( $0.7 \%$ in Fig. 2) can produce a majority $c(4 \times 2)$ reconstruction even at RT.

Our data also reveals that the energy separation between the $\pi$ and $\pi^{*}$ dangling bond surface states is almost the same for the $\mathrm{Ge}(001)[\sim 0.65 \mathrm{eV}]$ and $\mathrm{Si}(001)[\sim 0.70 \mathrm{eV}]$ surfaces. This highlights the similarity of the dangling bond states of both surfaces. The electronic structure of these two surfaces near $E_{F}$ are quite different, however, as the energy gap for $\mathrm{Ge}(001)$ separates the highest energy occupied back bond surface states from the lowest energy unoccupied $\pi^{*}$ dangling bond states. This is because, in contrast to $\mathrm{Si}(001)$, the dangling bond and back bond surface states for $\mathrm{Ge}(001)$ are separated in energy. This energy separation $(0.35 \mathrm{eV})$ is large enough for the back bond states to be imaged separately from the dangling bond states in STM. Our results thus suggest that Ge(001) may, under certain conditions, behave very differently to $\operatorname{Si}(001)$.

In summary, we have used DFT and STM to study the valence electronic surface states of the clean $\mathrm{Ge}(001) c(4 \times$ 2) surface at room temperature. We have shown that the top of the valence band consists of the back bond surface states associated with the $\mathrm{Ge}$ dimer atoms, while the surface dangling bonds are $\sim 0.35 \mathrm{eV}$ lower in energy and partially in resonance with the bulk states. These results resolve previous ambiguities regarding the nature of the surface states for $\mathrm{Ge}(001)$.

This work was supported by the University of Newcastle, the Australian Research Council (grant No. DP0557331), and the Australian Partnership for Advanced Computing. S. R. S. acknowledges support of the Australian Government.

*marian.radny@newcastle.edu.au

${ }^{\dagger}$ Present address: London Centre for Nanotechnology, University College, London $\mathrm{WC} 1 \mathrm{H} \mathrm{OAH}$, United Kindgom.

[1] R. A. Wolkow, Annu. Rev. Phys. Chem. 50, 413 (1999).

[2] P. W. Loscutoff and S. F. Bent, Annu. Rev. Phys. Chem. 57, 467 (2006).

[3] H. J. W. Zandvliet, Phys. Rep. 388, 1 (2003).

[4] E. Landemark, R. G. Uhrberg, P. Kruger, and J. Pollmann, Surf. Sci. Lett. 236, L359 (1990).

[5] Y. Yoshimoto, Y. Nakamura, H. Kawai, M. Tsukada, and M. Nakayama, Phys. Rev. B 61, 1965 (2000).

[6] J. Jeon, C. C. Hwang, T.-H. Kang, K.-J. Kim, B. Kim, Y. Chung, and C. Y. Park, Phys. Rev. B 74, 125407 (2006).

[7] K. Nakatsuji, Y. Takagi, F. Komori, H. Kusuhara, and A. Ishii, Phys. Rev. B 72, 241308 (2005).

[8] O. Gurlu, H. J. W. Zandvliet, and B. Poelsma, Phys. Rev. Lett. 93, 066101 (2004).

[9] G. Kresse and J. Furthmuller, Comput. Mater. Sci. 6, 15 (1996).

[10] M. W. Radny, G. A. Shah, P. V. Smith, S. R. Schofield, and N. J. Curson, J. Chem. Phys. (to be published).

[11] R. Rossmann, H. L. Meyerheim, V. Jahns, J. Wever, W. Maritz, D. Wolf, D. Dornish, and H. Schulz, Surf. Sci. 279, 199 (1992).

[12] S. Ferrer, X. Torrelles, V. H. Etgens, H. A. van der Vegt, and P. Fajardo, Phys. Rev. Lett. 75, 1771 (1995).

[13] J. Tersoff and D. R. Hamann, Phys. Rev. B 31, 805 (1985).

[14] R. Hoffmann, Solids and Surfaces: A Chemist's View of Bonding in Extended Structures (VCH, New York, 1998). 